\title{
Präoperative Vorbereitung: Patient Blood Management - Was ist optimal?
}

Patrick Meybohm, Markus M. Müller, Kai Zacharowski

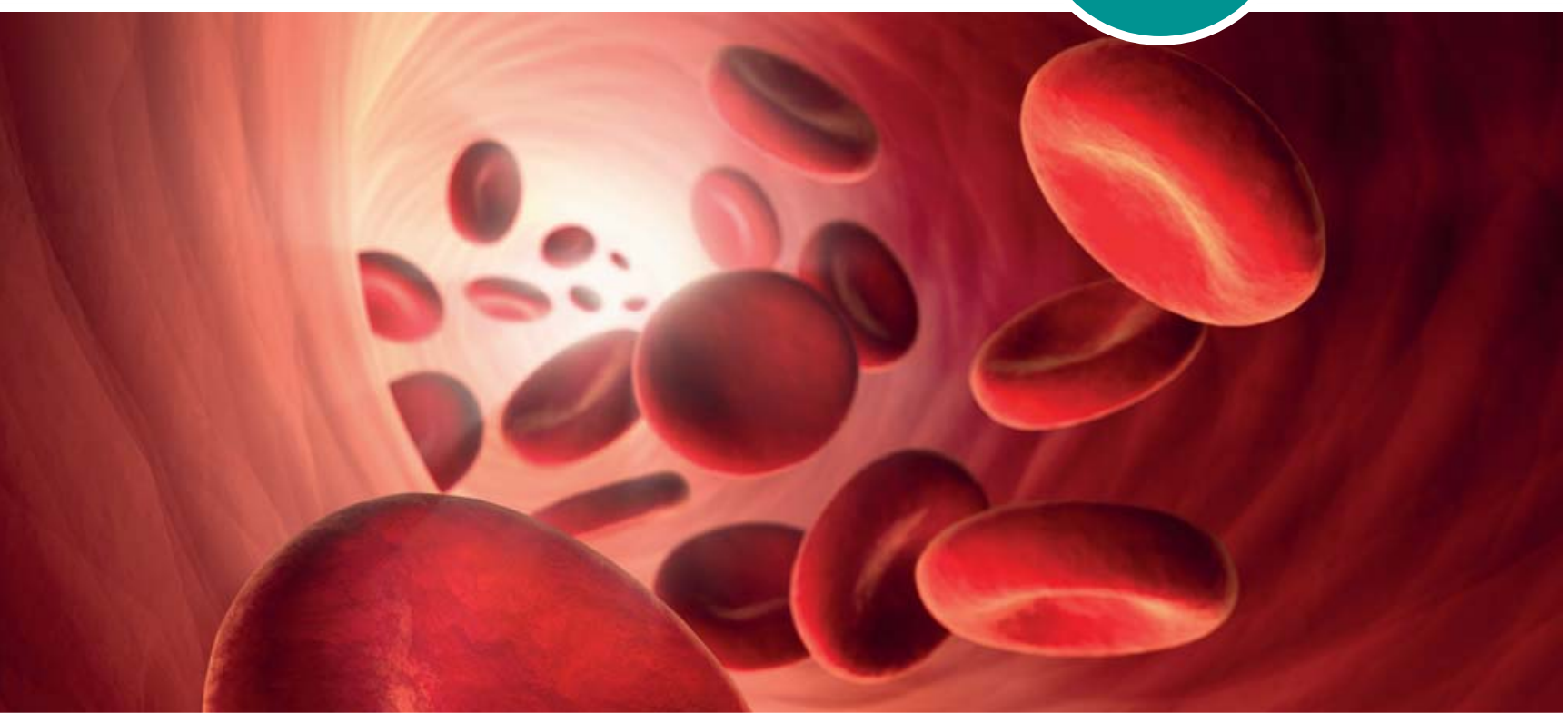

- Abb. 1 Quelle: psdesign1/Fotolia [rerif].

Die „Ressource Blut“ wird zunehmend knapper - das erfordert ihren rationalen Einsatz. Mit dieser Zielsetzung hat sich Patient Blood Management (PBM) in den letzten Jahren zu einem multimodalen, evidenzbasierten klinischen Behandlungsmodell entwickelt. Im Mittelpunkt dieses Beitrags stehen PBM-Komponenten der präoperativen Phase: Management einer Anämie, prätransfusionelle Vorbereitungen und präoperatives Management von Antikoagulanzien.

\section{PBM-Konzept}

Aufgrund medizinischer, gesellschaftlicher und ökonomischer Veränderungen wird Blut zu einer zunehmend knappen Ressource. Gleichzeitig stellt die Transfusion zellulärer Blutpräparate als „Transplantation des flüssigen Organs Blut" trotz Blutgruppenkompatibilität immer auch eine immunologische Herausforderung für den Empfängerorganismus dar. In diesem Sinn ist sie durchaus mit Risiken verbunden [1,2]. Vor diesem Hintergrund fordert die Weltgesundheitsorganisation seit 2011 die Einführung eines „Patient Blood Managements“-Konzepts [3]. Patient Blood Management (PBM) wurde inzwischen zu einem standardisierten, multimodalen, evidenzbasierten klinischen Behandlungsmodell mit mehr als
100 Einzelmaßnahmen entwickelt [4]. Die wesentlichen 3 Säulen sind aber

- ein umfassendes präoperatives Anämiemanagement,

- die Minimierung (unnötiger) iatrogener Blutverluste und

- die Ausschöpfung der natürlichen Anämietoleranz mit rationalem Einsatz von Erythrozytenkonzentrat(EK-)Transfusionen.

\section{Eine „optimale PBM-Ambulanz“}

Ein individueller präoperativer PBM-Plan startet idealerweise mit Indikationsstellung zur elektiven Operation. Für ein erfolgreiches präoperatives PBM-Programm sind verschiedene Voraussetzungen wichtig $[5,6]$ : 
- klare personelle Verantwortlichkeiten für das präoperative PBM-Programm - ein PBM-erfahrener Anästhesist kann hier als Ansprechpartner und Gatekeeper die Führung des PBM-Teams übernehmen,

- im Bedarfsfall ein erweitertes PBM-Team aus Spezialisten verschiedener Fachrichtungen, darunter Anästhesist, Chirurgen der verschiedenen Spezialgebiete, Transfusionsmediziner, Hämatologen, Gastroenterologen, um individuelle Problemlösungen aufzeigen zu können,

- ein gut kommuniziertes und geschultes PBM-Konzept, das für alle präoperativen Ambulanzen und das beteiligte pflegerische sowie ärztliche Personal als Arbeitsanweisung dient.

In Zukunft wäre eine engere Kooperation mit den zuweisenden (ambulanten) Kollegen wünschenswert. Wenn diesen der Wert des präoperativen PBM-Programms klar ist, können sie eine optimale Vorbereitung ihrer elektiven Patienten umsetzen.

Eine präoperative PBM-Ambulanz ist auf eine enge Verzahnung mit der chirurgischen und der anästhesiologischen Ambulanz angewiesen. Zum einen geht es darum, gemeinsam präoperative organisatorische und medizinische Herausforderungen zu lösen. Zum anderen rückt deutlich stärker bzw. zusätzlich der individuelle Patient in den Mittelpunkt der interdisziplinären Eingriffsplanung: die potenzielle präoperative Optimierung seines Erythrozytenvolumens und die Minimierung seines perioperativen Blutverlusts.

Eine langfristige Verpflichtung der Klinik und der Klinikleitung sowie des PBM-Teams auf die Umsetzung des PBMProgramms ist für eine nachhaltige Verankerung des PBM-Gedankens wichtig.

Merke

Ohne klar definierte Verantwortlichkeiten im PBMTeam, gute Kommunikation und Schulung aller innerund außerklinischen Beteiligten ist langfristig kein bleibender Erfolg des präoperativen PBM-Programms zu erwarten.

\section{Präoperatives Management der Anämie}

\section{Relevanz}

Entsprechend den Kriterien der WHO besteht eine Anämie, wenn der Hämoglobinwert bei Frauen unter $12 \mathrm{~g} / \mathrm{dl}$ und bei Männern unter $13 \mathrm{~g} / \mathrm{dl}$ liegt [7]. Musallam et al. berichten in einer Gesamtkohorte von 227425 stationären Patienten von einer Prävalenz der präoperativen Anämie von 30\% [8]. Im Krankenhaus ist die präoperative Anämie einer der stärksten Prädiktoren für die Gabe von EK während oder nach einer Operation. Außerdem ist eine präoperative Anämie - auch in milder Form - als eigenständiger und unabhängiger Risikofaktor für postoperative Komplikationen und eine erhöhte postoperative Sterblichkeit einzustufen [8-10].

Die Rationale für das präoperative Anämiekonzept ergibt sich somit aus

- der hohen Prävalenz der präoperativen Anämie und

- der medizinischen Notwendigkeit, diese - insbesondere vor elektiven Risikoeingriffen - zu diagnostizieren und so weit wie möglich vor der Operation zu behandeln.

Dabei spielen neben medizinischen und qualitätssichernden Aspekten auch betriebswirtschaftliche Überlegungen eine Rolle: Bei präoperativ anämischen Patienten kommt es zu längeren postoperativen Aufenthalten und erhöhten Komplikationsraten. Es erscheint daher geboten, einen Schwerpunkt der Qualitätssicherung im stationären sowie im ambulanten/prästationären Bereich auf PBM zu legen. Denn ein modernes präoperatives Anämiemanagement trägt zu einer höheren Patientensicherheit bei.

Merke

Eine präoperative Anämie ist häufig und mit signifikant erhöhter perioperativer Morbidität und Mortalität assoziiert. Jede Anämie sollte präoperativ abgeklärt und jeder nicht dringliche Eingriff bis nach der Anämiebehandlung - wenn medizinisch umsetzbar verschoben werden [11].

\section{Ursachen}

Die Ursachen der Anämie können angeboren sowie erworben sein und sind vielfältig [12-15]. Zu den Ursachen gehören u.a.:

- erhöhter Eisenbedarf (Schwangere, Blutspender, Leistungssport)

- Ernährung (Vegetarier, Veganer)

- Störung der enteralen Eisenresorption (Zöliakie, Helicobacter-pylori-Gastritis, Z.n. Gastrektomie, duodenaler Bypass/Magenbypass, atrophische Gastritis, chronisch entzündliche Darmerkrankungen wie Colitis ulcerosa, Morbus Crohn, genetische Faktoren)

- chronischer Blutverlust über den Magen-Darm-Trakt (Angiodysplasien, Neoplasien, Ulkus, Divertikel, Hämorrhoiden, Wurmerkrankungen)

- chronischer Blutverlust urogenital (Menorrhagie, Hämolyse)

- Medikamente (u.a. Antazida und Säureblocker [z. B. Pantoprazol], nicht steroidale Antirheumatika, Azetylsalizylsäure etc.)

- chronische Niereninsuffizienz und EPO-Therapie

- chronische Herzinsuffizienz (Resorption reduziert und Inflammation)

- Adipositas (chronische Inflammation)

- geriatrische Patienten (multifaktoriell) 


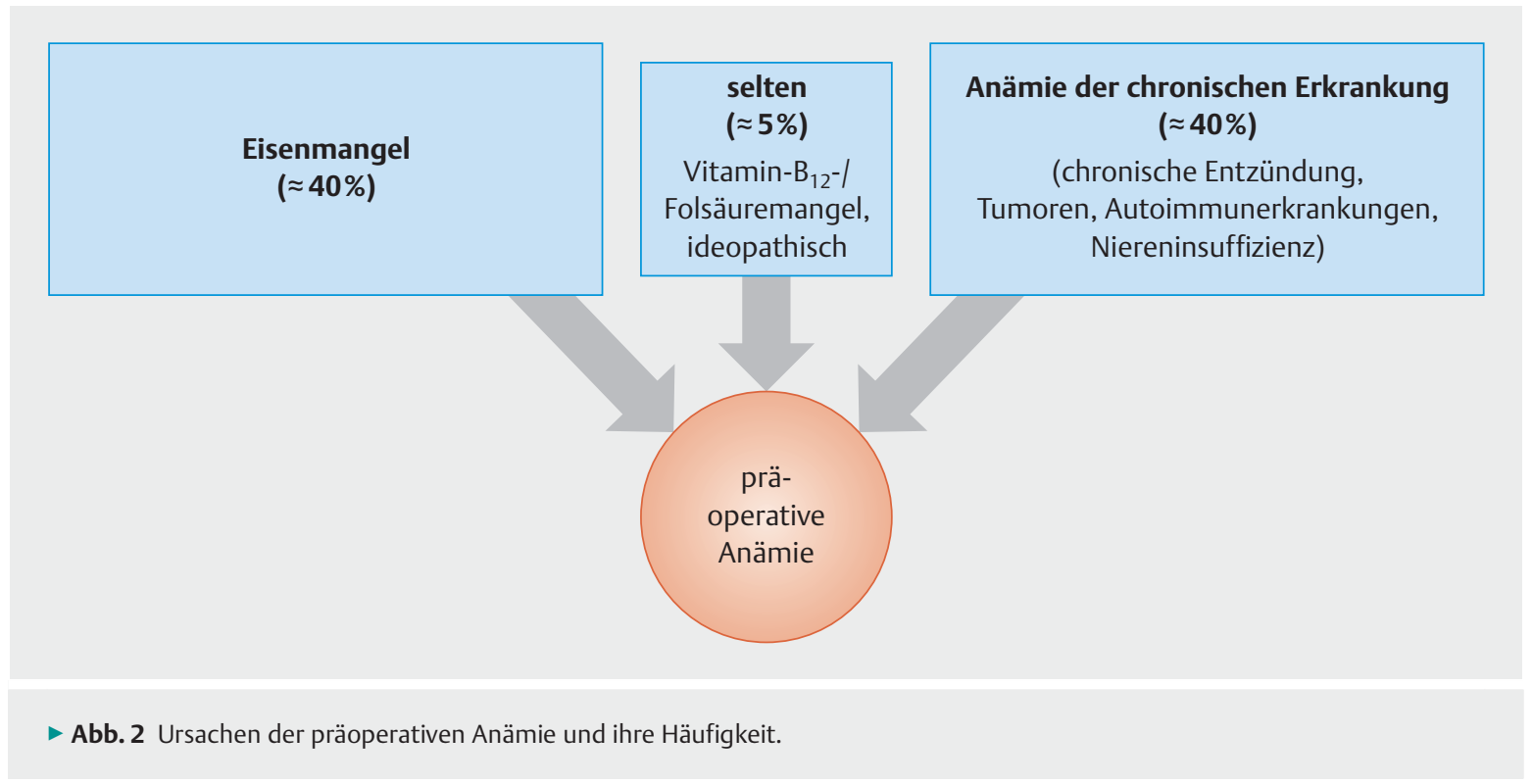

Für den praktischen Alltag vereinfacht dargestellt, liegt bei bis zu $40 \%$ der Patienten eine Eisenmangelanämie [12] vor. In ca. $40 \%$ besteht eine Anämie bei chronischen Erkrankungen (z.B. Tumoranämie, Autoimmunerkrankungen, Infektionen) und in ca. 5\% der Fälle eine seltenere Form ( $\triangleright$ Abb. 2).

\section{Differenzialdiagnosen}

Im Alltag sind die Symptome einer Anämie relativ unspezifisch und für den Patienten aufgrund natürlicher Kompensationsmechanismen in der Regel recht spät wahrnehmbar. Auftretende Konzentrationsstörungen und Ermüdungserscheinungen werden im Allgemeinen oft dem stressigen Alltag oder dem Alter zugeschrieben. Für die Differenzialdiagnose der Anämie können die in - Tab. 1 dargestellten Laborparameter genutzt werden $[11,16]$.

\section{Laborbefunde}

Die Basis bildet ein kleines Blutbild mit den Erythrozytenindizes MCV (mittleres Zellvolumen der Erythrozyten) und $\mathrm{MCH}$ (mittlerer Hämoglobingehalt der Erythrozyten). Daneben können je nach Anamnese, Befund und Vorbefunden auch Retikulozytenzahl (Retis) sowie Retikulozytenhämoglobin (Ret-He/CHr) für die Einordnung und das Management des Patienten bedeutungsvoll sein.

Beim Verdacht auf Eisenmangel sind Ferritin, Transferrinsättigung und - je nach Labormöglichkeiten - weitere Parameter wie der Anteil hypochromer Erythrozyten, lösliche Transferrinrezeptoren (sTfR) oder Zinkprotoporphyrin zu bestimmen. Bei Entzündung/Infektion, Autoimmunerkrankung, hepatozellulärer Erkrankung, Alkoholismus, Hypothyreose oder der Einnahme oraler Kontrazeptiva ist Ferritin als Akute-Phase-Protein bei einmaliger Be- stimmung ein schlechter Indikator. Dagegen ist sTfR bei Inflammation normal.

Besteht der Verdacht auf eine Nierenbeteiligung, so ist zumindest die Bestimmung von Kreatinin, Harnstoff und Elektrolyten, evtl. sogar der Kreatinin-Clearance, oder die Erythropoetin-Bestimmung sinnvoll.

Bei Verdacht auf eine Hämolyse, die immunologisch, medikamenteninduziert oder selten einmal auch physikalisch ausgelöst sein kann, eignen sich Haptoglobin, LDH und Bilirubin zur weiteren Klärung. Bei Verdacht auf eine antikörperinduzierte Hämolyse sollte immer auch transfusionsmedizinische Expertise zugezogen werden.

\section{Weiterführende Diagnostik}

In Abhängigkeit von den bis zu diesem Zeitpunkt erhobenen anamnestischen Daten und Untersuchungsbefunden kann aus dem PBM-Team heraus weitere Diagnostik veranlasst werden.

Einfache diagnostische Verfahren wie die Stuhluntersuchung auf okkultes Blut haben leider eine geringe Sensitivität und Spezifität. Bei Verdacht auf eine Anämieursache im Magen-Darm-Trakt kann die Diagnostik daneben auch auf endoskopische Verfahren wie Gastroskopie und Koloskopie sowie auf sonografische Verfahren ausgeweitet werden.

Eine weitere diagnostische Eskalation muss im Einzelfall vom zuständigen Facharztkollegen initiiert und im PBMTeam hinsichtlich der Eingriffsplanung besprochen werden. - Abb. 3 zeigt am Beispiel des Universitätsklinikums Frankfurt ein praktikables Vorgehen. 
- Tab. 1 Diagnostische Maßnahmen zur Eingrenzung der Ursache einer Anämie.

\begin{tabular}{|c|c|}
\hline Differenzialdiagnose & Laborbefunde und Untersuchungen \\
\hline $\begin{array}{l}\text { Verdacht auf Eisenmangel } \\
\text { (-Anämie) }\end{array}$ & 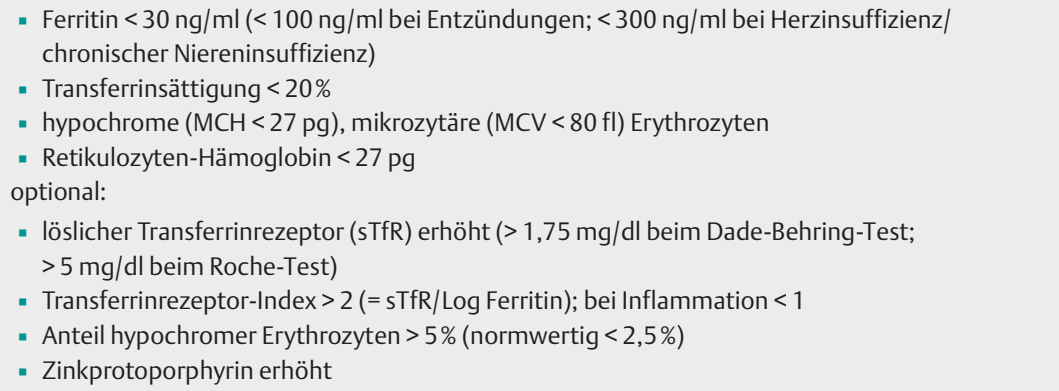 \\
\hline $\begin{array}{l}\text { Verdacht auf Vitamin- } B_{12}- \\
\text { Mangel, Folsäuremangel }\end{array}$ & $\begin{array}{l}\text { - Vitamin } B_{12}<200 \text { pg/ml (Norm: 197-866) } \\
\text { - Folsäure }<2 \text { ng/ml (Norm: 2,0-9,1) } \\
\text { - hyperchrome (MCH >34 pg), makrozytäre (MCV > } 98 \text { fl) Erythrozyten } \\
\text { - Medikamentenanamnese! } \\
\text { - Gastroskopie bei Vitamin-B12-Mangel; Intrinsic Factor (inkl. Antikörperbestimmung gegen } \\
\text { Parietalzellen, Intrinsic Factor, Schilddrüsenantigene) } \\
\text { - neurologische Untersuchung! }\end{array}$ \\
\hline Verdacht auf renale Anämie & $\begin{array}{l}\text { - Elektrolyte (Na, K, Ca, Cl etc.) } \\
\text { - Harnstoff, Kreatinin, ggf. Kreatinin-Clearance } \\
\text { " ggf. Erythropoetin-Bestimmung }\end{array}$ \\
\hline $\begin{array}{l}\text { Verdacht auf hämolytische } \\
\text { Anämie }\end{array}$ & $\begin{array}{l}\text { - } \text { direkter Coombs-Test: Nachweis von antikörperbeladenen Erythrozyten } \\
\text { - indirekter Coombs-Test (z. B. beim Antikörpersuchtest), Nachweis nicht gebundener, im Serum } \\
\text { zirkulierender antierythrozytärer Antikörper } \\
\text { - LDH erhöht } \\
\text { - Haptoglobin erniedrigt } \\
\text { - Fragmentozyten erhöht } \\
\text { - Medikamentenanamnese! } \\
\text { - indirektes Bilirubin erhöht } \\
\text { - Hämoglobinurie (bräunlicher Urin) } \\
\text { - je nach Vorbefunden auch transfusionsmedizinisches Konsil! }\end{array}$ \\
\hline $\begin{array}{l}\text { Verdacht auf chronische } \\
\text { (okkulte) GI-Blutung }\end{array}$ & $\begin{array}{l}\text { - Stuhluntersuchung auf okkultes Blut, ggf. auch sofort Gastroskopie bzw. Koloskopie, } \\
\text { ggf. radiologische Diagnostik }\end{array}$ \\
\hline $\begin{array}{l}\text { Verdacht auf gynäkologische/ } \\
\text { urologische Blutungsquelle }\end{array}$ & $\begin{array}{l}\text { - Vorstellung beim gynäkologischen Konsiliarius } \\
\text { - Urinuntersuchung auf Blut (Schnelltest), Vorstellung beim Urologen }\end{array}$ \\
\hline
\end{tabular}

Merke

Körperliche und Laboruntersuchungen - vor allem Parameter des Eisenstoffwechsels - sollen beim anämischen präoperativen Patienten die häufigsten Anämieursachen abklären. Je nach deren Ergebnissen folgen ggf. apparative und weitere Laboruntersuchungen.

\section{Therapie}

Eine präoperative Anämiekorrektur in enger Zusammenarbeit mit Hausärzten senkte in einer englischen Studie die Inzidenz einer präoperativen Anämie am OP-Tag von 26 auf $10 \%$. Auch die Notwendigkeit einer intraoperativen Fremdbluttransfusion wurde um die Hälfte von 26 auf $13 \%$ verringert [17].

Im deutschen Krankenhausalltag werden häufig Argumente gegen eine präoperative Behandlung der Anämie genannt: insbesondere Kosten, die Trennung von ambu- lanter und stationärer Versorgung sowie die potenzielle zeitliche Verschiebung des operativen Eingriffs. Doch im Vergleich zur Fremdbluttransfusion könnte sich die präoperative Anämiebehandlung lohnen [11,17-19]:

- hinsichtlich der Kosten,

- für den Patienten selbst (weniger transfusionsassoziierte Risiken und Nebenwirkungen),

- für die Klinik (Patientenrekrutierung und Marketing) als auch

- für die Allgemeinheit (Blutkonservenknappheit, schnellere Rehabilitation).

\section{Eisensubstitution}

Für den Fall, dass eine Eisenmangelanämie präoperativ diagnostiziert wurde, sollte ein Therapieintervall von idealerweise 2-4 Wochen eingeplant werden. Aus diesem Grund ist die Vorstellung des Patienten in der PBMAmbulanz zum frühestmöglichen Zeitpunkt wichtig. Dies gilt vor allem bei Eingriffen mit einer Transfusionswahr- 


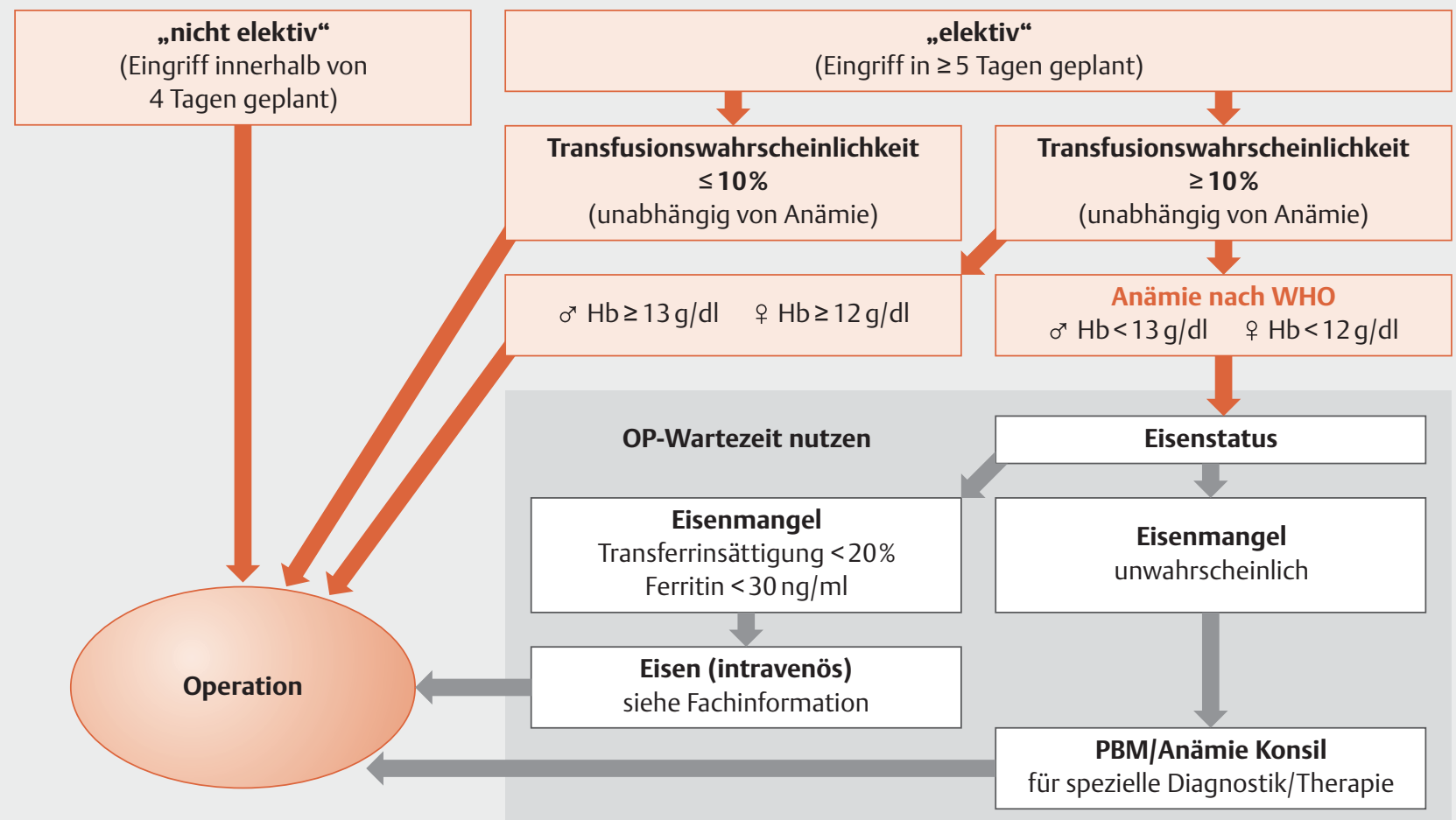

- Abb. 3 Algorithmus zum Vorgehen bei präoperativer Anämie bei elektiven und nicht elektiven Eingriffen (Daten des Universitätsklinikums Frankfurt).

scheinlichkeit von $>10 \%$ oder einem erwarteten Blutverlust von $>500 \mathrm{ml}$. In aller Regel macht die Dringlichkeit des Eingriffs (<6 Wochen) einen Behandlungsversuch mit oralen Eisenpräparationen unmöglich. Oft haben Patienten eine orale Eisensubstitution in der Vergangenheit wegen gastrointestinaler Probleme und Unverträglichkeiten abbrechen müssen. Es finden sich aber auch Berichte über mangelnde oder fehlende Effizienz der oralen Eisensubstitution trotz gesicherter Compliance.

Die Alternative sind parenterale Eisensubstitutionspräparate, die sich u.a. durch ihre Komplexstabilität voneinander unterscheiden [20-22]. Bei geringer Komplexstabilität werden vermehrt Eisenionen in der Blutbahn freigesetzt, bevor der Komplex von Makrophagen des mononukleären Phagozytensystems - insbesondere in Leber und Milz - phagozytiert werden kann. Solche freien Eisenionen im Blut können die Transportkapazität von Transferrin übersteigen und zu oxidativem Stress, Blutdruckabfall und Endothelschäden führen.

Bei der Etablierung des präoperativen PBM-Programms ist es deshalb wichtig, dass alle Entscheidungsträger gemeinsam festlegen, welche diagnostischen Wege gegangen werden und welche Therapeutika im Rahmen des präoperativen PBM-Programms verwendet werden sol- len. Es bieten sich hierzu neuere stabile i.v. Eisenpräparate an, mit denen im Rahmen einer einmaligen Gabe 5001500 mg Eisen substituiert werden können (s. „Fallbeispiel“). Ein häufig gehörtes Argument gegen Eisen i.v. ist ein erhöhtes Infektionsrisiko. Dies konnte in einer aktuellen Metaanalyse von 103 Studien (Zeitraum 19652013) mit 19253 Patienten jedoch nicht bestätigt werden [20]. Typische unerwünschte Arzneimittelwirkungen von Eisen i.v. können eher Übelkeit, Erbrechen, Juckreiz, Kopfschmerzen, Quaddeln und Erythem sein. Eine paravenöse Infusion von Eisen verursacht „lebenslang“ sichtbare Hautverfärbungen.

Im Oktober 2013 wurde ein Rote-Hand-Brief veröffentlicht, der auf schwere Überempfindlichkeitsreaktionen bei i.v. Eisenpräparaten hinweist [23]. Das Nutzen-Risiko-Verhältnis der i.v. Eisenpräparate aber wurde weiterhin positiv bewertet, vorausgesetzt die unter „Info“ genannten Empfehlungen [23] werden befolgt. 
- Tab. 2 Prätransfusionelle Analytik in Abhängigkeit von der Transfusionswahrscheinlichkeit.

\begin{tabular}{|l|l|}
$\begin{array}{l}\text { Transfusionswahrscheinlichkeit } \\
\text { (basierend auf hausinternen Daten) }\end{array}$ & Analytik \\
\hline$<1 \%$ (z. B. Hernienchirurgie) & keine Analytik \\
\hline $1-10 \%$ (z. B. Laparoskopie, primäre Gelenkendoprothetik) & Bestimmung von Blutgruppe, Antikörpersuchtest („Type \& Screen“) \\
\hline $\begin{array}{l}>10 \% \text { (z. B. Herzchirurgie, große Gefäßchirurgie, onkologi- } \\
\text { sche Viszeralchirurgie, Revisions-Endoprothetik) }\end{array}$ & $\begin{array}{l}\text { Bestimmung von Blutgruppe, Antikörpersuchtest, Kreuzprobe und } \\
\text { Bereitstellung (zentral oder in Satelliten-Depots) }\end{array}$ \\
\hline
\end{tabular}

\section{INFO}

Empfehlungen zu i.v. Eisenpräparaten

- Beachtung von Kontraindikation bei Überempfindlichkeit gegen den Wirkstoff

- Anwendung nur, wenn geschulte Fachkräfte sowie eine vollständige Ausrüstung zur Reanimation unverzüglich verfügbar sind

- Patientenüberwachung während und bis mindestens 30 min nach jeder i.v. Applikation

- Behandlung während der Schwangerschaft auf das 2. und 3. Trimenon begrenzen, sofern der Nutzen der Therapie eindeutig die potenziellen Risiken für die Mutter und den Fetus überwiegt

- Eisenpräparate zur i.v. Applikation sind bei Eisenmangelzuständen indiziert, wenn die orale Gabe unzureichend ist oder schlecht vertragen wird

Anmerkend muss noch hinzugefügt werden, dass diese Empfehlungen zwar für alle i.v. Eisenpräparate gelten, die Sicherheitsbedenken bezüglich des Risikos schwerer Überempfindlichkeitsreaktionen aber auf älteren (z. B. Eisendextran, -glukonat, -sucrose) und nicht auf den komplexeren Eisenpräparaten (z. B. Eisencarboxymaltose, -oxytol) beruhten.

Als Kontraindikation für eine parenterale Eisensubstitution gelten u. a. Überempfindlichkeit gegen den Wirkstoff, Hämochromatose, akute Infektionen, chronische Lebererkrankungen, myelodysplastisches Syndrom. Weitere Informationen müssen der jeweiligen Fachinformation entnommen werden.

\section{Folsäure/Vitamin-B12-Substitution}

Im Fall einer (eher selten) nachgewiesenen Anämie als Folge eines Mangels von Folsäure/Vitamin $B_{12}$ sollte eine entsprechende Substitution erfolgen:

- Folsäure (z. B. 10 mg p.o./d über 30 Tage)

- Vitamin B12 (z.B. $1000 \mu \mathrm{g} /$ Woche i.v./s.c. über 90 Tage)

\section{Erythropoese-stimulierende Agenzien}

Erythropoese-stimulierende Agenzien (ESA) wie Erythropoetin haben sicherlich einen ausgeprägten Stellenwert sowie eine Zulassung für die Behandlung renaler Anämien mit einem nachweisbaren Erythropoetin-Mangel. Auch im Rahmen der sehr selten indizierten präoperativen autologen Eigenblutspende spielen ESA eine Rolle: beispielsweise bei Patienten, die aufgrund komplexer antierythrozytärer Antikörpergemische sehr schwer mit Fremdblut zu versorgen sind. Inwieweit auch bei anderen Anämieformen der Einsatz von ESA gerechtfertigt ist, ist Gegenstand wissenschaftlicher Untersuchungen [2426]. In Deutschland fiele im präoperativen Setting der Einsatz von ESA (z. B. Epoetin alfa $2 \times 40000 \mathrm{IE}$ ) unter „OffLabel-Use“. Eine Ausnahme sind erwachsene Patienten ohne Eisenmangel vor großen orthopädischen Eingriffen.

Merke

Ein relevanter Anteil der Patienten mit einer präoperativen Anämie zeigt Potenzial für eine präoperative Anämietherapie. Die Indikation sollte so früh wie möglich gestellt werden.

\section{FALLBEISPIEL}

Eine 71-jährige Patientin wird von den Viszeralchirurgen um 10:30 Uhr zur Prämedikation in die Anästhesie-Ambulanz geschickt (geplante Whipple-OP in ca. 2-3 Wochen). Da die Wahrscheinlichkeit einer EK-Transfusion $>10 \%$ liegt ( $>$ Tab. 2), nimmt die Ambulanz-Arzthelferin ein kleines Blutbild, Eisenstatus sowie Routinelabor ab und sendet das Blut ins Zentrallabor. Während der Wartezeit auf das Anästhesiegespräch erfolgt die Analytik. Gegen Mittag liegen die Laborbefunde elektronisch vor: Hb 10,5 g/dl, Ferritin $20 \mathrm{ng} / \mathrm{ml}$ und Transferrinsättigung 8\%. Gemäß der WHO-Definition besteht ein Eisenmangel (Ferritin $<30 \mathrm{ng} / \mathrm{ml}$ ) mit einer Anämie $(\mathrm{Hb}<12 \mathrm{~g} / \mathrm{dl})$. Eine orale Eisensubstitution würde innerhalb von 3 Wochen kaum einen Effekt erzielen. Daher erhält die Patientin bei fehlenden Kontraindikationen (u. a. CRP 0,9 mg/dl) einmalig $1000 \mathrm{mg}$ Eisencarboxymaltose i.v. (in $100 \mathrm{ml} \mathrm{NaCl}$ ) über 20 min ohne besondere Auffälligkeiten. Sie wird währenddessen an einem Monitor mit 3-Kanal-EKG, nicht invasivem Blutdruck und peripherer Sauerstoffsättigung überwacht. Kurz vor 13:00 Uhr verlässt die Patientin zu Fuß die Anästhesie/Anämie-Ambulanz. Am OP-Tag 3 Wochen später zeigt sich in der 1. intraoperativen BGA ein Hb-Wert von $12,1 \mathrm{~g} / \mathrm{dl}$. Der intraoperative Blutverlust wird auf ungefähr $1100 \mathrm{ml}$ geschätzt. Die Patientin verbringt 1 Nacht im 24-h-Aufwachraum und kann am nächsten Tag hämodynamisch stabil mit einem HbWert von 8,6 g/dl ohne allogene Bluttransfusion auf die Normalstation verlegt werden. 


\section{Präoperative prätransfusionelle Vorbereitungen}

Zum PBM-Programm gehört neben einem Anämiemanagement zentral in Abhängigkeit vom Blutungs- und Transfusionsrisiko auch die möglichst frühzeitige Bestimmung von Blutgruppe und Antikörpersuchtest des Patienten [5]. Häufig sind die anämischen Patienten - vor allem, wenn präoperativ die Anämie nicht behandelt wird - perioperativ Kandidaten für eine allogene EK-Transfusion. Somit ist die Kenntnis der Blutgruppe des Patienten für die Operationsplanung erforderlich. Darüber hinaus ist es wichtig zu wissen, ob ein klinisch relevanter antierythrozytärer Antikörper im Plasma vorliegt. Denn diese Antikörper erschweren eine ggf. notwendige und dringliche Transfusion aufgrund des dann nötigen Zeitaufwands für die Auswahl der Präparate.

\section{Kreuzprobe/Bereitstellung}

Nur wenn die Transfusionswahrscheinlichkeit nach den hauseigenen Daten über $10 \%$ liegt ( $\bullet$ Tab. 2), müssen EK präoperativ gekreuzt und bereitgestellt werden. Der Patient ist zudem über Transfusionsalternativen (v. a. Anämiemanagement, autologe Hämotherapieverfahren wie Hämodilution und maschinelle Autotransfusion) aufzuklären. Wichtig ist in diesem Zusammenhang, dass vor der Operation eine aktuelle Kreuzprobe und ein aktueller Antikörpersuchtest vorliegen müssen. Beide sind 3 Tage (plus Abnahmetag) nach Blutentnahme gültig. Der Antikörpersuchtest muss also ggf. kurzfristig vor dem Operationstermin wiederholt werden. Kreuzproben und EK-Bereitstellung sollten erst bei diesem Blutabnahmetermin kurz vor Operation erfolgen.

\section{Merke}

Aktuelle hausinterne Daten sollten berücksichtigt werden. Eine Kreuzprobe mit Bereitstellung von EK soll nur für Eingriffe mit einer Transfusionswahrscheinlichkeit $>10 \%$ erfolgen. Das vermeidet unnötige Analytik und schont Fremdblutressourcen.

\section{Blutgruppe und Antikörpersuchtest}

Es ist sinnvoll, bei den Beratungen der Transfusionskommission des Krankenhauses das Vorgehen für die operativen Eingriffe mit einer Transfusionswahrscheinlichkeit von 1 bis 10\% festzulegen. Das heißt, es gilt zu entscheiden, ob hier eine Blutgruppenbestimmung und ein aktueller Antikörpersuchtest präoperativ durchzuführen sind - das sog. „Type \& Screen“-Verfahren.

Vorteile dieses Verfahrens sind, dass bei einer unerwarteten Blutungskomplikation

- die Blutgruppe des Patienten bereits bekannt ist,

- ein negativer Antikörpersuchtest auch gefahrlos eine Notfalltransfusion blutgruppengleich mit ungekreuz- ten EK (und Nachkreuzen, sobald der Notfall dies erlaubt) ermöglicht und

- aktuelles Blut des Patienten bereits im Labor für die Kreuzproben vorliegt - falls ein kleiner Zeitpuffer dafür vorhanden ist.

Auch im Fall eines präoperativ positiven Antikörpersuchtests bleibt genügend Zeit, die Diagnostik durchzuführen und ggf. sogar die Operation zu verschieben, bis ausreichend kompatible EK für den Patienten bereitstehen ( Tab. 2).

Merke

Bei Patienten mit vorbekannten antierythrozytären Alloantikörpern soll präoperativ Rücksprache mit dem verantwortlichen Labor gehalten werden.

\section{Präoperatives Management von Antikoagulanzien}

\section{Individuelle Risikostratifizierung}

Etwa 1-3\% der Bevölkerung erhalten zur Verhinderung arterieller und venöser Thromboembolien oder nach Stentintervention dauerhaft eine Antikoagulation. Hierbei können je nach Indikation u.a. Heparine, (direkte) orale Antikoagulanzien oder Thrombozytenaggregationshemmer zum Einsatz kommen. Eine unkritische Einnahme der Medikamente bis zum OP-Tag würde mit einem sehr hohen Blutungsrisiko und einer intra-/postoperativen Koagulopathie einhergehen. Ein generelles frühzeitiges Absetzen der Medikation wäre aber genauso unverantwortlich, da in diesem Fall Thromboembolien oder Stentverschlüsse drohen würden [27].

Um für den Patienten sowohl das Blutungs- als auch das Thromboembolierisiko zu minimieren, hat daher in der präoperativen PBM-Ambulanz die Medikamentenanamnese eine besondere Bedeutung. Dabei sollten sowohl Gerinnungs- und Thrombozytenfunktionshemmer, u.a. aber auch Medikamente mit Einfluss auf den Eisenstoffwechsel (z. B. Säureblocker und Antazida) aktiv abgefragt werden $[28,29]$. Hierbei ist auch Wert auf nicht verschreibungspflichtige Arzneimittel zu legen, die vielen Patienten als „unwichtig“ erscheinen, z. B. nicht steroidale Entzündungshemmer (NSAID).

Eine frühzeitige individuelle Risikostratifizierung sollte folgende Faktoren berücksichtigen [30]:

- Indikation für die Antikoagulation

- Risikoprofil für Thrombose und Blutung

- Komorbidität (Nieren, Leber, Knochenmark)

- Komedikation (Plättchenaggregationshemmer, nicht steroidale Antirheumatika)

- Dringlichkeit der Operation

- Regionalanästhesie 
- Blutungsrisiko durch die geplante Operation (gering/ moderat/hoch)

- Wechsel der Antikoagulation (Bridging/Switching)

\section{Merke}

Eine sorgfältige Medikamentenanamnese und eine individuelle Risikostratifizierung sind die wichtigsten Voraussetzungen für das perioperative Management von Antikoagulanzien.

\section{Direkte orale Antikoagulanzien}

Aktuell werden direkte orale Antikoagulanzien (DOAK) bei folgenden 5 Indikationen eingesetzt:

- Thromboseprophylaxe bei elektivem Hüft- oder Kniegelenkersatz

- Therapie und Sekundärprophylaxe der tiefen Venenthrombose

- Schlaganfallprophylaxe bei nicht valvulärem Vorhofflimmern

- akutes Koronarsyndrom

- Lungenarterienembolie

Dabei stellen die Patienten mit Vorhofflimmern die mit Abstand größte Gruppe dar. Bei Patienten mit Vorhofflimmern erfolgt die Risikostratifizierung für Schlaganfall/Thromboembolien mithilfe des $\mathrm{CHA}_{2} \mathrm{DS}_{2}$-VAScScores ( $\triangleright$ Tab. $\mathbf{3})$.

\section{INFO}

Wirkmechanismus der DOAK

Die Wirkung der DOAK basiert auf der direkten Hemmung der aktiven Zentren der Gerinnungsenzyme, wie Faktor Xa (Rivaroxaban, Apixaban, Edoxaban) oder Thrombin (Dabigatran). Durch die hohe Bindungsaffinität direkt an das aktive Zentrum der Enzyme wird die Substratumsetzung verhindert. Die Wirkung der DOAK setzt daher sofort nach Einnahme und Resorption ein. Die Wirkung ist reversibel und hat eine dem niedermolekularen Heparin (NMH) vergleichbar kurze Halbwertszeit. Die Zulassungsstudien zeigten für DOAK gegenüber den klassischen oralen Antikoagulanzien mindestens einen vergleichbaren klinischen Effekt zur Prävention der venösen Thrombose/des ischämischen Schlaganfalls.

\section{Vor- und Nachteile der DOAK}

Die Vorteile der DOAK liegen vor allem bei weniger schweren Blutungskomplikationen (z.B. intrakranielle Blutung), schnellerem Wirkeintritt, kürzerer Halbwertszeit und damit besserer Steuerbarkeit mit weniger Monitoring.

Patientenindividuelle Faktoren haben allerdings Einfluss auf die Pharmakokinetik. So wurde eine zunehmende Va-
- Tab. 3 Schlaganfallrisikoabschätzung bei Patienten mit Vorhofflimmern nach dem $\mathrm{CHA}_{2} \mathrm{DS}_{2}$-VASc-Score (maximal 9 Punkte).

\begin{tabular}{|l|l|l|}
\hline Buchstabe & Bedeutung & Punkte \\
\hline C & chronische Herzinsuffizienz & 1 \\
\hline H & arterielle Hypertonie & 1 \\
\hline $\mathrm{A}_{2}$ & Alter $\geq 75$ Jahre & 2 \\
\hline D & Diabetes mellitus & 1 \\
\hline S $_{2}$ & $\begin{array}{l}\text { vorangegangener Schlag- } \\
\text { anfall, transitorische ischä- } \\
\text { mische Attacke, Embolie }\end{array}$ & 2 \\
\hline V & $\begin{array}{l}\text { periphere arterielle Ver- } \\
\text { schlusskrankheit }\end{array}$ & 1 \\
\hline A & Alter 65 bis 74 Jahre & 1 \\
\hline Sc & weibliches Geschlecht & 1 \\
\hline $\begin{array}{l}\text { 2 } 2 \text { Punkte: hohes Schlaganfallrisiko } \rightarrow \text { orale Antikoagulation } \\
\text { empfohlen }\end{array}$ & \\
\hline
\end{tabular}

riabilität vor allem bei Patienten in hohem Alter, mit eingeschränkter Nierenfunktion, eingeschränkter Leberfunktion, Unter- und Übergewicht sowie Komedikation mit Plättchenaggregationshemmern beschrieben. Die aktuell publizierten Empfehlungen zum prä- und perioperativen Vorgehen unter DOAK sind teilweise widersprüchlich. Viele Empfehlungen stammen aus konservativen Fachrichtungen, wo die perioperative Problematik nur unzureichend abgebildet wird.

Niedriges Blutungsrisiko

(unabhängig vom Thromboembolierisiko)

- Abb. 4 beschreibt das Management für DOAK bei Eingriffen mit niedrigem Blutungsrisiko. Dies sind Eingriffe, bei denen chirurgische Blutungen selten sind, oder Eingriffe, bei denen chirurgische Blutungen aufgrund ihrer Lokalisation gut beherrschbar sind, z.B. Zahnbehandlung, Dermatologie. Hier ist in der Regel aufgrund der kurzen Halbwertszeit keine Überbrückungstherapie in der perioperativen Phase erforderlich. Die notwendige Therapiepause kann durch einfaches Weglassen vor OP erreicht werden.

\section{Hohes Blutungs- und niedriges/mittleres Thromboembolierisiko}

Verschiedene Komorbiditäten (z.B. eingeschränkte Nierenfunktion, eingeschränkte Leberfunktion) und Komedikationen (z.B. zusätzliche Antikoagulanzien) können trotz einer Therapiepause von 2-facher Halbwertszeit mit einer relevanten Blutungsneigung assoziiert sein. Daher muss bei Eingriffen mit hohem Blutungsrisiko die gerinnungshemmende Therapie mit DOAK frühzeitiger pausiert werden (5-fache Halbwertszeit, > Abb. 5). Dies sind Eingriffe, bei denen eine klinisch signifikante Blutung nicht ausgeschlossen werden kann: große Bauch- oder Gefäßoperationen, große orthopädische Operationen, 


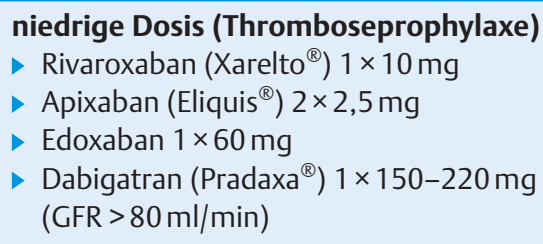

- Rivaroxaban (Xarelto $\left.{ }^{\circledR}\right) 1 \times 10 \mathrm{mg}$

- Apixaban (Eliquis ${ }^{\circledR}$ ) $2 \times 2,5 \mathrm{mg}$

- Edoxaban $1 \times 60 \mathrm{mg}$

- Dabigatran (Pradaxa ${ }^{\circledR}$ ) $1 \times 150-220 \mathrm{mg}$ (GFR $>80 \mathrm{ml} / \mathrm{min})$

volle Dosis (therapeutische Antikoagulation)

- Rivaroxaban $1 \times 20 \mathrm{mg}$

- Apixaban $2 \times 5 \mathrm{mg}$

- Edoxaban $1 \times 60 \mathrm{mg}$

\section{Regionalanästhesie \\ - Rivaroxaban $1 \times 10-20 \mathrm{mg}$ \\ - Apixaban $2 \times 2,5-5 \mathrm{mg}$ \\ - Edoxaban $1 \times 60 \mathrm{mg}$}

\section{Dabigatran $1 \times 150-220 \mathrm{mg}$ (GFR $<80 \mathrm{ml} / \mathrm{min}$ )}

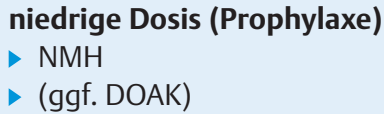

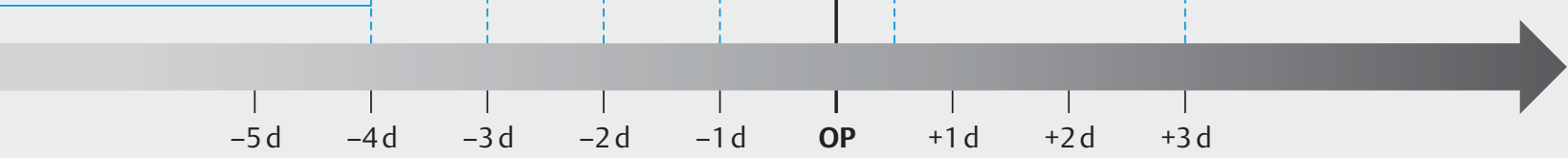

bei fehlender Blutungsneigung postoperativ Neustart mit niedriger Dosis so bald als möglich (aber nicht $<6-8 \mathrm{~h}$ )

- Abb. 4 Perioperatives Management für direkte orale Antikoagulanzien (DOAK) bei niedrigem Blutungsrisiko (unabhängig vom Thromboembolierisiko) (Vorgehen am Universitätsklinikum Frankfurt). NMH: niedermolekulares Heparin

große intrathorakale Eingriffe, Punktion nicht komprimierbarer Gefäße.

\section{Maximales Blutungsrisiko (unabhängig vom Thromboembolierisiko)/Hohes Blutungsrisiko \& hohes Thromboembolierisiko}

Liegt ein maximales Blutungsrisiko (unabhängig vom Thromboembolierisiko) oder ein hohes Blutungsrisiko und ein hohes Thromboembolierisiko vor, so sollte ein echtes "Switching“ erfolgen ( $\mathbf{A} \mathbf{A b} \mathbf{b} . \mathbf{6})$.

- maximales Blutungsrisiko (unabhängig vom Thromboembolierisiko): Eingriffe an „kritischen Organen“ (Neurochirurgie, intraokulär), bei denen bereits kleine Blutungen großen Schaden verursachen können,

- hohes Blutungsrisiko und hohes Thromboembolierisiko:

- Eingriffe, bei denen eine klinisch signifikante Blutung nicht ausgeschlossen werden kann: große Bauchoperationen, große Gefäßoperationen, große orthopädische Operationen, große intrathorakale Eingriffe, Punktion nicht komprimierbarer Gefäße und

- hohes Thromboembolierisiko, z. B. $\mathrm{CHA}_{2} \mathrm{DS}_{2}$-VAScScore $>5$, venöse Thromboembolie $<3$ Monate.

\section{Merke}

Das perioperative Management der direkten oralen Antikoagulanzien richtet sich nach dem Blutungsrisiko des geplanten chirurgischen Eingriffs und dem individuellen Thromboembolierisiko des Patienten.

\section{Umgang mit Thrombozytenaggregations- hemmern}

\section{Azetylsalizylsäure}

Die aktuellen Leitlinien der European Society of Cardiology empfehlen grundsätzlich die perioperative Weitergabe von Azetylsalizylsäure (ASS) im Rahmen der Sekundärprävention [27]. Insbesondere bei negativer Blutungsanamnese stellt die Monotherapie mit ASS $100 \mathrm{mg}$ i.d.R. keine Kontraindikation für chirurgische Eingriffe dar.

Bei intrakraniellen Eingriffen, Operationen am Spinalkanal oder Augenhintergrund ist das Absetzen der Thrombozytenaggregationshemmer aufgrund des hohen Blutungsrisikos jedoch erforderlich. Hierbei sollte ASS $100 \mathrm{mg} 7$ Tage präoperativ pausiert werden. 
niedrige Dosis (Thromboseprophylaxe)

- Rivaroxaban (Xarelto $\left.{ }^{\circledR}\right) 1 \times 10 \mathrm{mg}$

- Apixaban (Eliquis ${ }^{\circledR}$ ) $2 \times 2,5 \mathrm{mg}$

- Edoxaban $1 \times 60 \mathrm{mg}$

- Dabigatran $\left(\right.$ Pradaxa $\left.^{\circledR}\right) 1 \times 150-220 \mathrm{mg}$ (GFR $>80 \mathrm{ml} / \mathrm{min}$ )

volle Dosis (therapeutische Antikoagulation)

- Rivaroxaban $1 \times 20 \mathrm{mg}$

- Apixaban $2 \times 5 \mathrm{mg}$

- Edoxaban $1 \times 60 \mathrm{mg}$

\section{Regionalanästhesie}

- Rivaroxaban $1 \times 10-20 \mathrm{mg}$

- Apixaban $2 \times 2,5-5 \mathrm{mg}$

- Edoxaban $1 \times 60 \mathrm{mg}$

\section{Dabigatran $1 \times 150-220 \mathrm{mg}$}

(GFR $<80 \mathrm{ml} / \mathrm{min})$ niedrige Dosis (Prophylaxe)

- $\mathrm{NMH}$

(ggf. DOAK)

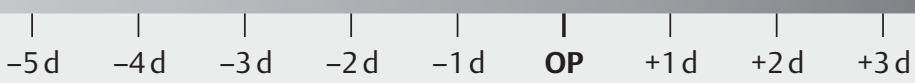

\section{volle Dosis (therapeu- tische Antikoagulation) \\ - DOAK \\ - $\mathrm{NMH}$}

Rivaroxaban $\left(\right.$ Xarelto $^{\circledR}$ )

Apixaban (Eliquis ${ }^{\circledR}$ )

Edoxaban $\left(\right.$ Lixiana $\left.^{\circledR}\right)$

Dabigatran (Pradaxa ${ }^{\circledR}$ )

bei fehlender Blutungsneigung postoperativ Neustart mit niedriger Dosis so bald als möglich (aber nicht $<6-8 \mathrm{~h}$ )

- Abb. 5 Perioperatives Management für direkte orale Antikoagulanzien (DOAK) bei hohem Blutungsrisiko und niedrigem/mittlerem Thromboembolierisiko (Vorgehen am Universitätsklinikum Frankfurt). NMH: niedermolekulares Heparin

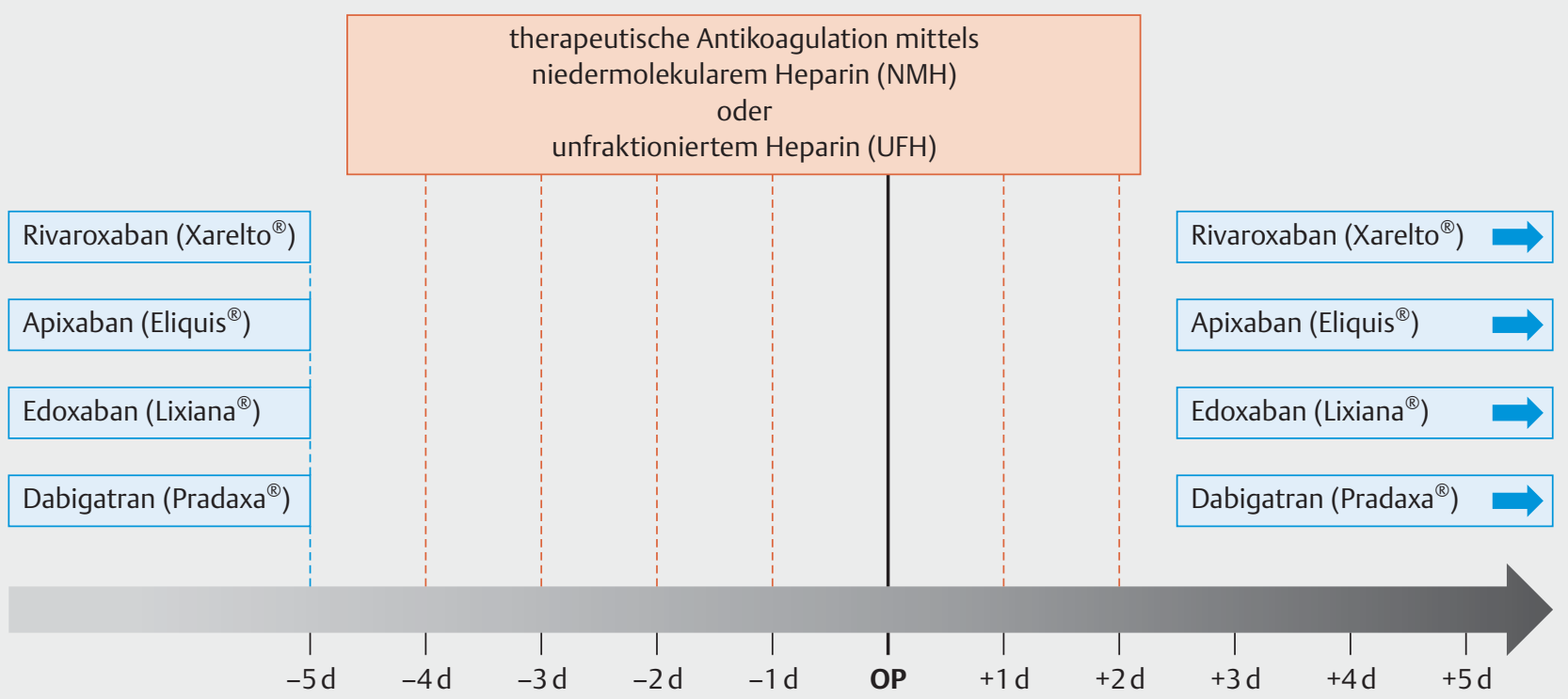

- Abb. 6 Perioperatives Management für direkte orale Antikoagulanzien (DOAK) bei maximalem Blutungsrisiko (unabhängig vom Thromboembolierisiko)/hohem Blutungsrisiko und hohem Thromboembolierisiko (Vorgehen am Universitätsklinikum Frankfurt). 


\section{FALLBEISPIEL}

Ein 63-jähriger Patient erhält wegen paroxysmalen Vorhofflimmerns und Z. n. Beckenvenenthrombose $2 \times 5$ mg Apixaban. Wegen eines akuten Koronarsyndroms und Implantation von 3 Drug-eluting Stents (DES) vor ca. 3 Monaten steht er nun zusätzlich unter dualer Thrombozytenhemmung (100 mg ASS, 75 mg Clopidogrel). Seit 2 Wochen berichtet der Patient zunehmend Schwindel, Kopfschmerz und Doppelbilder. Im cCT zum Ausschluss einer intrakraniellen Blutung und dem nachfolgenden cMRT stellt sich der Verdacht auf eine raumfordernde zerebelläre Metastase, die dringlich neurochirurgisch entfernt werden soll. Nach DES-Implantation und nicht valvulärem Vorhofflimmern wäre eine Tripletherapie für 6 Monate indiziert, danach duale Therapie mit oraler Antikoagulation plus 1 Thrombozytenhemmer für weitere 6 Monate. Das perioperative Management muss nun 2 Faktoren berücksichtigen: ein hohes Thromboembolierisiko und ein maximales Blutungsrisiko. Die Antikoagulation mit Apixaban wird 5 Tage präoperativ abgesetzt und nach 1 Tag Unterbrechung mit einem NMH (Clexane $2 \times 40 \mathrm{mg}$ s. c.) bis $24 \mathrm{~h}$ präoperativ fortgeführt. Auch Clopidogrel wird 5 Tage vor OP ausgesetzt, ASS am Tag vor der OP. Die OP verläuft problemlos, bei diffuser Blutungsneigung werden am OP-Ende 2 Thrombozytenkonzentrate transfundiert. Postoperativ wird bei fehlender Blutungsneigung die Antikoagulation langsam gesteigert: $10 \mathrm{~h}$ postoperativ Clexane $1 \times 20 \mathrm{mg} ; 1$. Tag Clexane $1 \times 40 \mathrm{mg} ; 2$. Tag ASS $100 \mathrm{mg}+$ Clexane $2 \times 40 \mathrm{mg} ; 3$. Tag ASS/Clexane $2 \times 60$ mg; ab 10. Tag ASS/Clopidogrel/Clexane; nach der Rehaklinik Umstellung auf Tripletherapie inkl. Apixaban.

\section{Duale Plättchenhemmung -}

ASS und P2Y12-Rezeptorinhibitor

Präoperativ sollte bei stabilen Patienten nur ASS als Monotherapie bis zur OP fortgeführt werden. Die duale Plättchenhemmung mit Gabe von ADP-Rezeptor-Blockern sollte 5-7 Tage präoperativ pausiert werden:

- Clopidogrel/Ticagrelor: 5 Tage

- Prasugrel: 7 Tage

Ausnahmen stellen Hochrisikopatienten nach Stentimplantation dar, bei denen eine Kombinationstherapie (duale Plättchenhemmung) indiziert ist. In dieser Patientengruppe ist das jährliche Wiederholungsrisiko eines atherothrombotischen Verschlusses bzw. einer Stentthrombose nach Absetzen der Therapie hoch bis sehr hoch. Daher muss die kombinierte Gabe von ASS und P2Y12-Rezeptorinhibitoren mindestens für folgende Zeiträume beibehalten werden:

\section{- 4 Wochen nach einem Bare Metal Stent (BMS)}

- 6 Monate nach einem Drug-eluting Stent (DES)

Ist ein operativer Eingriff in diesem Zeitraum zwingend notwendig, sollte dieser in der kritischen Phase nach Möglichkeit immer mit dualer Plättchenhemmung durchgeführt werden. Die Leitlinie der European Society of Cardiology 2012 besagt: Ein Pausieren des P2Y12-Rezeptorinhibitors wäre bei einem „Bridging“ durch kontinuierlich i.v. kurz wirksames Tirofiban oder Eptifibatid zur reversiblen Plättchenhemmung möglich. Hierzu liegen aber keine Studien vor und die praktische Umsetzung präoperativ auf einer Normalstation ist eher schwierig. Vor diesem Hintergrund bietet sich auch das sog. „inverse Bridging“ an. Hierbei wird die duale Plättchenhemmung als aktive Antikoagulation bis zum Tag vor der Operation fortgeführt. Am OP-Tag erfolgt vor dem Hautschnitt die Transfusion von 2 Thrombozytenkonzentraten. Postoperativ wird ASS dann nach $6 \mathrm{~h}$ wieder angesetzt, um bei diesen Hochrisikopatienten die Phase der inadäquaten Thrombozytenhemmung so kurz wie möglich zu halten.

Im Fall einer therapiebedürftigen perioperativen Koagulopathie sollten (weitere) Thrombozytenkonzentrate unter Überwachung der Thrombozytenfunktion (z. B. Impedanzaggregometrie) transfundiert werden.

\section{Cave}

Aktive Metabolite von Prasugrel, und auch Clopidogrel können bei intra- oder postoperativer Blutung auch substituierte Thrombozyten hemmen!

\section{Fazit}

Das Hauptaugenmerk eines präoperativen PBM-Programms liegt darauf, patienteneigene Ressourcen zu schonen und zu stärken durch

- präoperatives Erkennen und Therapie einer Anämie,

- risikoadaptierte prätransfusionelle Diagnostik sowie

- abgestuftes präoperatives Management der Antikoagulanzien unter Berücksichtigung des individuellen Risikoprofils von Blutung und Thromboembolie. 


\section{KERNAUSSAGEN}

- Hauptaufgabe eines präoperativen PBM-Programms ist es, patienteneigene Ressourcen zu schonen und zu stärken.

- Das gelingt mit einem individuell adaptierten professionellen Management der Anämie, prätransfusionellen Vorbereitungen sowie Management von Antikoagulanzien.

- Eine präoperative Anämie, auch in milder Form, ist ein eigenständiger und unabhängiger Risikofaktor für postoperative Komplikationen und eine erhöhte postoperative Sterblichkeit.

- Prinzipiell sollte man jede Anämie präoperativ abklären und nicht dringliche Eingriffe bis zum Abschluss der entsprechenden Anämiebehandlung bei behandelbaren Ursachen verschieben.

- Ein großer Teil der Patienten mit präoperativer Anämie zeigt Potenzial für eine präoperative Anämietherapie (z. B. parenterale Substitution von stabilen Eisenkomplexen). Die Indikation sollte so früh wie möglich gestellt werden.

- Die präoperative prätransfusionelle Analytik sollte aktuelle hausinterne Daten berücksichtigen. Die Diagnostik richtet sich nach der Transfusionswahrscheinlichkeit des Eingriffs:

- < $1 \%$ : keine Diagnostik

- 1-10\%: nur Bestimmung der Blutgruppe und Antikörpersuchtest (Type \& Screen-Konzept)

- >10\% eine Kreuzprobe mit Bereitstellung von EK

- Weder eine unkritische Antikoagulation bis zum OP-Tag (hohes Blutungsrisiko) noch ein generelles frühzeitiges Absetzen (Risiko für Thromboembolien oder Stentverschlüsse) ist adäquat. Vielmehr muss das präoperative Management von Antikoagulanzien unter Berücksichtigung des individuellen Risikoprofils von perioperativer Blutung und Thromboembolie erfolgen.

- Ohne klar definierte Verantwortlichkeiten im PBMTeam, gute Kommunikation und Schulung aller inner- und außerklinischen Beteiligten kann sich langfristig kein bleibender Erfolg des präoperativen PBM-Programms einstellen.

Interessenkonflikt

P. M. und K.Z. erhielten finanzielle Förderungen von B. Braun Melsungen, CSL Behring, Fresenius Kabi und Vifor Pharma für eine Investigator-initiierte Studie zur Implementierung des Patient Blood Management Programms in vier Universitätsklinika.

P.M. und/oder K.Z. erhielten Förderungen oder Reisekostenunterstützung für Beratungen und Vorträge der folgenden Firmen: Abbott GmbH \& Co. KG, AesculapAkademie GmbH, AQAI GmbH, AstellasPharma GmbH, AstraZeneca GmbH, Aventis Pharma GmbH, B. Braun Melsungen AG, Baxter
Deutschland GmbH, Biosyn GmbH, Biotest AG, Bristol-Myers Squibb GmbH, CSL Behring GmbH, Dr. F. KöhlerChemie GmbH, Dräger Medical GmbH, Essex Pharma GmbH, Fresenius Kabi GmbH, Fresenius Medical Care, Gambro Hospal GmbH, Gilead, GlaxoSmithKline $\mathrm{GmbH}$, Grünenthal $\mathrm{GmbH}$, Hamilton Medical AG, HCCM Consulting GmbH, Heinen + Löwenstein $\mathrm{GmbH}$, Janssen-Cilag $\mathrm{GmbH}$, med Update $\mathrm{GmbH}$, Medivance EU B. V., MSD Sharp \& Dohme GmbH, Novartis Pharma GmbH, Novo Nordisk Pharma GmbH, P. J. Dahlhausen \& Co. GmbH, Pfizer Pharma GmbH, Pulsion Medical Systems S. E., Siemens Healthcare, Teflex Medical GmbH, Teva GmbH, TopMedMedizintechnik GmbH, Verathon Medical, ViforPharma $\mathrm{GmbH}$. M. M. M. gibt keine weiteren Interessenskonflikte an.

Über die Autoren

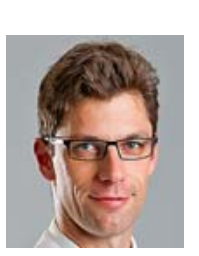

\section{Patrick Meybohm}

Prof. Dr. med., Leitender Oberarzt für die Bereiche Anästhesiologie und Operative Intensivmedizin am Universitätsklinikum Frankfurt am Main. Klinische und wissenschaftliche Schwerpunkte: Patient Blood Management, Intensivmedizin, Immunsystem und Gerinnung, perioperatives Outcome und Patientensicherheit.

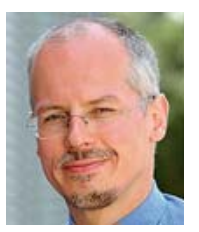

\section{Markus M. Müller}

Dr. med., Facharzt für Transfusionsmedizin, Hämostaseologie. Oberarzt und Abteilungsleiter Blutentnahme (Außendienst) am Institut für Transfusionsmedizin und Immunhämatologie des DRK-Blutspendediensts Baden-Württemberg/Hessen in Frankfurt, Universitätsklinikum Frankfurt am Main. Darüber hinaus ist er als einer von 6 Facharztkollegen für das MVZ DRK-Blutspendedienst tätig.

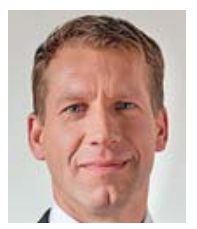

\section{Kai Zacharowski}

Prof. Dr. Dr. med., Direktor der Klinik für Anästhesiologie, Intensivmedizin und Schmerztherapie des Universitätsklinikums Frankfurt am Main. Klinische und wissenschaftliche Schwerpunkte: Patient Blood Management, Intensivmedizin, Immunsystem und Gerinnung, perioperatives Outcome und Patientensicherheit.

\section{Korrespondenzadresse}

Prof. Dr. med. Patrick Meybohm

Klinik für Anästhesiologie, Intensivmedizin und Schmerztherapie

Universitätsklinikum Frankfurt am Main

Theodor-Stern-Kai 7

60590 Frankfurt am Main

patrick.meybohm@kgu.de

\section{Literatur}

[1] Rohde JM, Dimcheff DE, Blumberg N et al. Health care-associated infection after red blood cell transfusion: a systematic review and meta-analysis. JAMA 2014; 311: 1317-1326

[2] Anthes E. Evidence-based medicine: Save blood, save lives. Nature 2015; 520: 24-26 
[3] Sixty-third World Health Assembly. Resolution on availability, safety and quality of blood products (WHA 63.12). 2010. Im Internet: http://www.who.int/bloodsafety/transfusion_services/self_sufficiency/en/; Stand: 14.03.2017

[4] Meybohm P, Richards T, Isbister J et al. Patient blood management bundles to facilitate implementation. Transfus Med Rev 2017; 31: 62-71

[5] Muller MM, Fischer D, Stock U et al. [Patient blood management - The preoperative patient]. Anasthesiol Intensivmed Notfallmed Schmerzther 2014; 49: 246-253; quiz 254

[6] Muller MM, Meybohm P, Geisen C et al. [Patient blood management - How does it work in practice? - the interdisciplinary cooperation]. Anasthesiol Intensivmed Notfallmed Schmerzther 2014; 49: 266-272

[7] World Health Organization. Nutritional Anemia: Report of a WHO Scientific Group. World Health Organ Tech Rep Ser 1968; 405: 5-37

[8] Musallam KM, Tamim HM, Richards T et al. Preoperative anaemia and postoperative outcomes in non-cardiac surgery: a retrospective cohort study. Lancet 2011; 378: 1396-1407

[9] von Heymann C, Kaufner L, Sander M et al. Does the severity of preoperative anemia or blood transfusion have a stronger impact on long-term survival after cardiac surgery? J Thorac Cardiovasc Surg 2016; 152: 1412-1420

[10] Leichtle SW, Mouawad NJ, Lampman R et al. Does preoperative anemia adversely affect colon and rectal surgery outcomes? J Am Coll Surg 2011; 212: 187-194

[11] Goodnough LT, Maniatis A, Earnshaw P et al. Detection, evaluation, and management of preoperative anaemia in the elective orthopaedic surgical patient: NATA guidelines. $\mathrm{Br}$ J Anaesth 2011; 106: 13-22

[12] Camaschella C. Iron-deficiency anemia. N Engl J Med 2015; 372: $1832-1843$

[13] Goodnough LT, Schrier SL. Evaluation and management of anemia in the elderly. Am J Hematol 2014; 89: 88-96

[14] Kassebaum NJ, Jasrasaria R, Naghavi M et al. A systematic analysis of global anemia burden from 1990 to 2010. Blood 2014; 123: 615-624

[15] Weiss G, Goodnough LT. Anemia of chronic disease. N Engl J Med 2005; 352: 1011-1023

[16] Retter A, Wyncoll D, Pearse R et al.; British Committee for Standards in Haematology. Guidelines on the management of anaemia and red cell transfusion in adult critically ill patients. Br J Haematol 2013; 160: 445-464

[17] Kotze A, Carter LA, Scally AJ. Effect of a patient blood management programme on preoperative anaemia, transfusion rate, and outcome after primary hip or knee arthroplasty: a quality improvement cycle. Br J Anaesth 2012; 108: 943-952

[18] Bisbe E, Molto L, Arroyo R et al. Randomized trial comparing ferric carboxymaltose vs. oral ferrous glycine sulphate for postoperative anaemia after total knee arthroplasty. Br J Anaesth 2014; 113: 402-409

[19] Johansson PI, Rasmussen AS, Thomsen LL. Intravenous iron isomaltoside $1000\left(\right.$ Monofer $^{\circledR}$ ) reduces postoperative anaemia in preoperatively non-anaemic patients undergoing elective or subacute coronary artery bypass graft, valve replacement or a combination thereof: a randomized double-blind placebocontrolled clinical trial (the PROTECT trial). Vox Sang 2015; 109: 257-266
[20] Avni T, Bieber A, Grossman A et al. The safety of intravenous iron preparations: systematic review and meta-analysis. Mayo Clin Proc 2015; 90: 12-23

[21] Geiser P. The pharmacology and safety profile of ferric carboxymaltose (Ferinject ${ }^{\circledR}$ ): structure/reactivity relationships of iron preparations. Port J Nephrol Hypert 2009; 23: 11-16

[22] Munoz M, Gomez-Ramirez S, Cuenca J et al. Very-short-term perioperative intravenous iron administration and postoperative outcome in major orthopedic surgery: a pooled analysis of observational data from 2547 patients. Transfusion 2014; 54: 289-299

[23] Die Hersteller von Eisen-Präparaten zur intravenösen Applikation. Rote-Hand-Brief zu Eisen-Präparaten zur intravenösen Applikation. Oktober 2013. Im Internet: http://www.akdae. de/Arzneimittelsicherheit/RHB/Archiv/2013/20131021.pdf; Stand: 15.03 .2017

[24] So-Osman C, Nelissen RG, Koopman-van Gemert AW et al. Patient blood management in elective total hip- and knee-replacement surgery (Part 1): a randomized controlled trial on erythropoietin and blood salvage as transfusion alternatives using a restrictive transfusion policy in erythropoietin-eligible patients. Anesthesiology 2014; 120: 839-851

[25] Voorn VM, van der Hout A, So-Osman C et al. Erythropoietin to reduce allogeneic red blood cell transfusion in patients undergoing total hip or knee arthroplasty. Vox Sang 2016; 111: 219-225

[26] Weltert L, Rondinelli B, Bello R et al. A single dose of erythropoietin reduces perioperative transfusions in cardiac surgery: results of a prospective single-blind randomized controlled trial. Transfusion 2015; 55: 1644-1654

[27] Kristensen SD, Knuuti J, Saraste A et al.; Authors/Task Force Members. 2014 ESC/ESA Guidelines on non-cardiac surgery: cardiovascular assessment and management: The Joint Task Force on non-cardiac surgery: cardiovascular assessment and management of the European Society of Cardiology (ESC) and the European Society of Anaesthesiology (ESA). Eur J Anaesthesiol 2014; 31: 517-573

[28] Nowak-Gottl U, Langer F, Limperger $V$ et al. [Bridging: Perioperative management of chronic anticoagulation or antiplatelet therapy]. Dtsch Med Wochenschr 2014; 139: 13011306

[29] Patel JP, Arya R. The current status of bridging anticoagulation. Br J Haematol 2014; 164: 619-629

[30] Giebl A, Gürtler K. [New oral anticoagulants in perioperative medicine]. Anaesthesist 2014; 63: 347-362

\section{Bibliografie}

DOI https://doi.org/10.1055/s-0042-108925

Anästhesiol Intensivmed Notfallmed Schmerzther 2017; 52: 326-340 @ Georg Thieme Verlag KG Stuttgart · New York ISSN 0939-2661 


\section{Punkte sammeln auf CME.thieme.de}

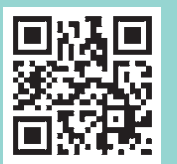

Diese Fortbildungseinheit ist 12 Monate online für die Teilnahme verfügbar.

Sollten Sie Fragen zur Online-Teilnahme haben, finden Sie unter cme.thieme.de/hilfe eine ausführliche Anleitung. Wir wünschen viel Erfolg beim Beantworten der Fragen!

Unter eref.thieme.de/ZZWHCDS oder über den QR-Code kommen Sie direkt zum Artikel zur Eingabe der Antworten.

VNR 2760512017152371565

\section{Frage 1}

Welche der folgenden Voraussetzungen ist für ein erfolgreiches präoperatives PBM-Programm am wenigsten wichtig?

A enge interdisziplinäre Kooperation, u. a. mit Chirurgen, Internisten, Transfusionsmedizinern

$B$ enge Kooperation mit den niedergelassenen Zuweisern

C gut kommunizierter und akzeptierter präoperativer PBMPlan

D Algorithmus für Massivblutung

$\mathrm{E}$ ein gut kommuniziertes und geschultes PBM-Konzept, das für alle präoperativen Ambulanzen und das beteiligte pflegerische sowie ärztliche Personal als Arbeitsanweisung dient

\section{Frage 2}

Welche Aussage zur präoperativen Anämie ist richtig?

A Die präoperative Anämie ist eher selten (weniger als 5\% der Fälle).

B Zur Abklärung einer präoperativen Anämie sind aufwendige und teure Messmethoden notwendig.

C Eisenmangel stellt eine häufige, leicht behandelbare Ursache einer präoperativen Anämie dar.

D Die Behandlung der präoperativen Anämie ist sinnlos, da der Patient durch die Anämie für die Operation bereits präkonditioniert ist und die Risiken der Anämie besser verträgt.

E Da die Transfusion von Erythrozytenkonzentraten am schnellsten den Hämoglobinwert anhebt, ist sie 1. Wahl zur Therapie der präoperativen Anämie.

\section{Frage 3}

Welche Aussage zur präoperativen prätransfusionellen immunhämatologischen Diagnostik ist falsch?

A Sie sollte bei Verdacht auf eine antikörperinduzierte Hämolyse unter Angabe dieses Verdachts eingeleitet werden.

B Sie ist nicht notwendig, weil sie postoperativ aussagekräftiger ist.

C Sie umfasst bei Patienten mit einer Transfusionswahrscheinlichkeit von 1-10\% Blutgruppenbestimmung und Antikörpersuchtest.

D Sie umfasst bei Patienten mit einer Transfusionswahrscheinlichkeit > 10\% die Blutgruppenbestimmung, den Antikörpersuchtest und die Bereitstellung gekreuzter Erythrozytenkonzentrate.

E Sie dient neben der Blutgruppenbestimmung dem Ausschluss bzw. der Bestimmung antierythrozytärer Antikörper.

\section{Frage 4}

Was gehört nicht zu den typischen Ursachen für eine Anämie?

A Eisenmangel

B niedriger Body-Mass-Index aufgrund einer Mangelernährung

C Inflammation

D chronische Krankheiten

E Polycythaemia vera

\section{Frage 5}

Welche der folgenden Maßnahmen ist in der präoperativen Phase nicht bei jedem chirurgischen Patienten erforderlich?

A Prüfung der Notwendigkeit für Thrombozytenaggregationshemmer

B sorgfältige Gerinnungs- und Medikamentenanamnese

C Prüfung der Notwendigkeit von oralen Antikoagulanzien

D Bestimmung von Blutgruppe, Antikörpersuchtest, Kreuzproben und Bereitstellung von Erythrozytenkonzentraten

E Abklärung einer präoperativen Anämie, vor allem bei erwartetem Blutverlust von $>500 \mathrm{ml}$

\section{- Weitere Fragen auf der folgenden Seite...}




\section{Punkte sammeln auf CME.thieme.de}

Fortsetzung...

\section{Frage 6}

Wann könnten Erythropoetin-Rezeptor-stimulierende Substanzen bei Patienten mit einer Anämie am ehesten von Nutzen sein?
A bei Mangelernährung
B bei chronisch entzündlichen Erkrankungen
$C$ bei Eisenmangel
D bei renaler Anämie
E bei hämolytischer Anämie

\section{Frage 7}

Welche Maßnahme sollte in der Regel nicht zur Optimierung der Erythropoese präoperativ eingesetzt werden?

A Diagnose und Behandlung einer Anämie

B Einbeziehung Erythropoese-stimulierender Substanzen

C Einbeziehung von intravenöser Eisensubstitution

D präoperative Bluttransfusion

E Einbeziehung von Folsäure oder Vitamin $B_{12}$

\section{Frage 8}

Was gehört nicht zu den Vorteilen einer präoperativen Behandlung einer Anämie im Vergleich zur allogenen Bluttransfusion?

A Eine Anämiebehandlung ist für den Patienten medizinisch von Nutzen.

B Eine Anämiebehandlung kann für Patient und Krankenhaus kostengünstiger sein.

C Die präoperative Anämiebehandlung ist effektiver und schneller im Vergleich zur allogenen Bluttransfusion.

D Die präoperative Substitution von intravenösem Eisen steigert bei Eisenmangelanämie den Hämoglobinwert innerhalb von 2-4 Wochen in der Regel um 1-2 g/dl.

E Eine präoperative Anämiebehandlung reduziert die Wahrscheinlichkeit einer perioperativen Transfusion von Erythrozytenkonzentraten.

\section{Frage 9}

Welche Aussage zu den direkten oralen Antikoagulanzien (DOAK) ist falsch?

A Zu den typischen Indikationen gehört die Thromboseprophylaxe bei elektivem Hüft- oder Kniegelenkersatz.

B Zu den typischen Indikationen gehört die Therapie und Sekundärprophylaxe der tiefen Venenthrombose.

C Zu den typischen Indikationen gehört die Schlaganfallprophylaxe bei nicht valvulärem Vorhofflimmern.

D Die Risikostratifizierung für Schlaganfall/Thromboembolien bei Vorhofflimmern erfolgt mithilfe des $\mathrm{CHA}_{2} \mathrm{DS}_{2}$-VAScScores.

E Ein typischer Vertreter der DOAK ist Warfarin.

\section{Frage 10}

Welche Aussage zum Management der DOAK ist falsch?

A Aufgrund der kurzen Halbwertszeit von DOAK kann bei niedrigem Blutungsrisiko und niedrigem Thromboembolierisiko die Gabe bis 1 Tag präoperativ erfolgen, und es ist keine Überbrückungstherapie in der perioperativen Phase erforderlich.

B Die Schlaganfallrisikoabschätzung bei Patienten mit Vorhofflimmern sollte nach dem Revised Cardiac Risk Index erfolgen.

C Bei Eingriffen mit hohem Blutungsrisiko sollte die gerinnungshemmende Therapie frühzeitiger pausiert werden (5fache Halbwertszeit).

D Bei Eingriffen mit einem maximalen Blutungsrisiko (kritische Organe, bei denen bereits kleine Blutungen großen Schaden verursachen können, z. B. Neurochirurgie, intraokulär) sollte ein Switching auf eine therapeutische Antikoagulation mittels niedermolekularem Heparin/unfraktioniertem Heparin erfolgen.

E Bei Eingriffen mit hohem Blutungsrisiko und zeitgleich hohem Thromboembolierisiko sollte ein Switching auf eine therapeutische Antikoagulation mittels niedermolekularem Heparin/unfraktioniertem Heparin erfolgen. 